\title{
UNIFORM PROJECTION DESIGNS
}

\author{
By FASHENG Sun ${ }^{1}$, YAPING WANG ${ }^{2}$ AND HongQuAN XU ${ }^{3}$ \\ Northeast Normal University, East China Normal University and \\ University of California, Los Angeles
}

\begin{abstract}
Efficient designs are in high demand in practice for both computer and physical experiments. Existing designs (such as maximin distance designs and uniform designs) may have bad low-dimensional projections, which is undesirable when only a few factors are active. We propose a new design criterion, called uniform projection criterion, by focusing on projection uniformity. Uniform projection designs generated under the new criterion scatter points uniformly in all dimensions and have good space-filling properties in terms of distance, uniformity and orthogonality. We show that the new criterion is a function of the pairwise $L_{1}$-distances between the rows, so that the new criterion can be computed at no more cost than a design criterion that ignores projection properties. We develop some theoretical results and show that maximin $L_{1}$-equidistant designs are uniform projection designs. In addition, a class of asymptotically optimal uniform projection designs based on good lattice point sets are constructed. We further illustrate an application of uniform projection designs via a multidrug combination experiment.
\end{abstract}

1. Introduction. Computer experiments are becoming ubiquitous in science, engineering and service for studying complex phenomena [Fang, Li and Sudjianto (2006), Santner, Williams and Notz (2003)]. Latin hypercube designs (LHDs) are popular in computer experiments because they can achieve the maximum onedimensional stratification. An LHD may have bad projections onto two or higher dimensions, and thus improvements of space-filling properties are needed based on other criteria. Orthogonality [Owen (1994), Tang (1998), Ye (1998)] and maximin distance criterion [Johnson, Moore and Ylvisaker (1990), Zhou and Xu (2014)] are two most commonly used design criteria. A number of authors have constructed orthogonal and maximin LHDs; see Lin and Tang (2015) for an excellent review, as well as Sun and Tang (2017), Xiao and Xu (2017, 2018) and Wang, Xiao and $\mathrm{Xu}$ (2018) for some recent works. However, as Joseph and Hung (2008) and Xiao and Xu (2018) pointed out, orthogonal LHDs often do not have maximin distance

Received January 2018; revised March 2018.

${ }^{1}$ Supported by NSFC Grant 11471069, Fundamental Research Funds for the Central Universities Grant and China Scholarship Council.

${ }^{2}$ Supported by China Scholarship Council.

${ }^{3}$ Supported by NSF Grant DMS-14-07560.

MSC2010 subject classifications. 62K99, 62K15.

Key words and phrases. Computer experiment, discrepancy, Latin hypercube design, maximin distance design, space-filling design, uniform design. 
and maximin distance designs are often not orthogonal, except for some specific cases [Wang, Yang and Xu (2018)].

Uniform designs are another class of space-filling designs which are widely used in both physical and computer experiments; see Fang et al. (2000), Liang, Fang and $\mathrm{Xu}$ (2001) and Fang, Li and Sudjianto (2006) for applications in dynamic systems, chemistry and chemical engineering and computer experiments. The main idea of uniform designs is to spread the design points uniformly over the entire design space. Many criteria have been proposed to measure the uniformity, among which the centered $L_{2}$-discrepancy is the most popular. Uniform designs with low discrepancy are model robust in the sense that they can guard against inaccurate estimates caused by model misspecification [Hickernell and Liu (2002)].

A computer or physical experiment often involves a large number of factors at an early stage among which only a few of them are active [Moon, Dean and Santner (2012), Woods and Lewis (2016), Kleijnen (2017)]. Therefore, space-filling designs with good projection properties are desirable for factor screening [Moon, Dean and Santner (2011), Joseph, Gul and Ba (2015)]. Maximin distance designs and uniform designs are space-filling in high dimensions, but can have bad lowdimensional projections. To address this problem, Moon, Dean and Santner (2011) proposed a two-dimensional distance metric and developed algorithms to construct designs with good projection properties. Joseph, Gul and Ba (2015) proposed another distance metric and constructed maximum projection designs.

We propose a new criterion, called uniform projection criterion, by focusing on two-dimensional projection uniformity. Our new criterion is based on the centered $L_{2}$-discrepancy, and the basic idea can be extended to other commonly used discrepancies. Uniform projection designs scatter points uniformly in all dimensions and have good space-filling properties in terms of distance, uniformity and orthogonality. This is an important feature as experimenters often do not know in advance which factors turn out to be important before conducting the experiment. We present examples to show that uniform projection designs have better projection properties than maximum projection designs constructed by Joseph, Gul and $\mathrm{Ba}$ (2015). In addition, we show that the new criterion is a function of the pairwise $L_{1}$-distances between the rows. It is well known that uniformity is closely related to orthogonality and aberration; see Fang and Mukerjee (2000), Ma, Fang and Lin (2003), Tang, $\mathrm{Xu}$ and Lin (2012) and Zhou and Xu (2014). The connection between projection uniformity and $L_{1}$-distance is surprising and important. This connection enables us to compute the new criterion at no more cost than a design criterion that ignores projection properties. The two-dimensional distance metric proposed by Moon, Dean and Santner (2011) does not have this advantage. In addition, this connection enables us to develop some general theoretical results. We show that maximin $L_{1}$-equidistant designs are uniform projection designs and construct a class of uniform projection designs based on good lattice point sets when the number of rows is an odd prime. We further provide an application of uniform projection designs to a multidrug combination experiment. 
This paper is organized as follows. Section 2 introduces some notation and definitions. Section 3 introduces the new design criterion. Section 4 studies projection properties of the new criterion and compares uniform projection designs with other existing designs. Section 5 derives some general theoretical results. We establish a connection between the new criterion and $L_{1}$-distance distribution and then derive a lower bound. We show in Theorem 3 that maximin $L_{1}$-equidistant designs are uniform projection designs and further construct a class of uniform projection designs based on good lattice points. Section 6 considers applications of uniform projection designs. We illustrate how uniform projection designs can be used to efficiently study drug combination experiments. Section 7 gives some concluding remarks. For clarity, the proofs are deferred to the Appendix.

2. Notation and definitions. Let $\left(n, s^{m}\right)$ denote a design with $n$ runs and $m$ factors, each taking $s$ levels from $\mathcal{Z}_{s}=\{0,1, \ldots, s-1\}$. Such a design can be represented by a matrix $D=\left(x_{i k}\right)_{n \times m}$. Throughout this paper, we only consider balanced designs where each level appears equally often in every column. An LHD is a balanced design with $s=n$.

For an $\left(n, s^{m}\right)$ design $D=\left(x_{i k}\right)$, let $\rho_{\text {ave }}(D)=\sum_{j \neq k}\left|\rho_{j k}\right| /[m(m-1)]$ be the average of absolute correlations between columns, where $\rho_{j k}$ is the correlation between the $j$ th and $k$ th columns. A design is orthogonal if $\rho_{\text {ave }}(D)=0$.

The $L_{p}$-distance between the $i$ th row $x_{i}=\left(x_{i 1}, \ldots, x_{i m}\right)$ and the $j$ th row $x_{j}=$ $\left(x_{j 1}, \ldots, x_{j m}\right)$ is

$$
d_{p}\left(x_{i}, x_{j}\right)=\left(\sum_{k=1}^{m}\left|x_{i k}-x_{j k}\right|^{p}\right)^{1 / p} .
$$

The $L_{p}$-distance of $D$ is $d_{p}(D)=\min \left\{d_{p}\left(x_{i}, x_{j}\right), 1 \leq i<j \leq n\right\}$. A maximin $L_{p^{-}}$ distance design maximizes the $d_{p}(D)$ value. Joseph, Gul and Ba (2015) proposed the following distance metric

$$
\psi(D)=\left\{\frac{1}{\left(\begin{array}{c}
n \\
2
\end{array}\right)} \sum_{i=1}^{n-1} \sum_{j=i+1}^{n} \frac{1}{\prod_{k=1}^{m}\left(x_{i k}-x_{j k}\right)^{2}}\right\}^{1 / m} .
$$

A maximum projection design minimizes the $\psi(D)$ value.

For an $\left(n, s^{m}\right)$ design $D=\left(x_{i k}\right)$, its (squared) centered $L_{2}$-discrepancy is defined as

$$
\begin{aligned}
\mathrm{CD}(D)= & \frac{1}{n^{2}} \sum_{i=1}^{n} \sum_{j=1}^{n} \prod_{k=1}^{m}\left(1+\frac{1}{2}\left|z_{i k}\right|+\frac{1}{2}\left|z_{j k}\right|-\frac{1}{2}\left|z_{i k}-z_{j k}\right|\right) \\
& -\frac{2}{n} \sum_{i=1}^{n} \prod_{k=1}^{m}\left(1+\frac{1}{2}\left|z_{i k}\right|-\frac{1}{2}\left|z_{i k}\right|^{2}\right)+\left(\frac{13}{12}\right)^{m},
\end{aligned}
$$

where $z_{i k}=\left(2 x_{i k}-s+1\right) /(2 s)$. 
3. Uniform projection designs. Designs with low discrepancy have good uniformity in the full-dimensional space, but can have bad projections in lowerdimensional spaces, which is undesirable when only a few factors are active. Since in practice two-factor interactions are more important than three-factor or higherorder interactions, we propose a new criterion focusing on two-dimensional projections. The new criterion, called uniform projection criterion, is defined as follows:

$$
\phi(D)=\frac{2}{m(m-1)} \sum_{|u|=2} \operatorname{CD}\left(D_{u}\right),
$$

where $u$ is a subset of $\{1,2, \ldots, m\},|u|$ denotes the cardinality of $u$ and $D_{u}$ is the projected design of $D$ onto dimensions indexed by the elements of $u$. The $\phi(D)$ defined in (3.1) is the average CD values of all two-dimensional projections of $D$. When $D$ has more than two factors, $\phi(D)$ is different from $\operatorname{CD}(D)$; see Example 1. A design achieving the minimum $\phi(D)$ value is a uniform projection design.

EXAmple 1. Consider four $25 \times 3$ LHDs in Table 1 . Design $D_{1}$ is a uniform design under the centered $L_{2}$-discrepancy from the uniform design website at Hong Kong Baptist University. Designs $D_{2}$ and $D_{3}$ are maximin distance design and maximum projection design constructed via R packages SLHD [Ba, Myers and Brenneman (2015)] and MaxPro [Joseph, Gul and Ba (2015)], respectively. We ran $\mathrm{R}$ commands maximinSLHD (with slice parameter $t=1$ ) and MaxProLHD 100 times with default settings and chose the best designs. The uniform projection design (UPD) $D_{4}$ is constructed via a threshold accepting algorithm [see Fang et al. (2000)] using the $\phi(D)$ criterion. Table 2 compares the centered $L_{2}$-discrepancy (CD), the $L_{2}$-distance $d_{2}(D), \psi(D)$ defined in (2.2), the uniform projection crite-

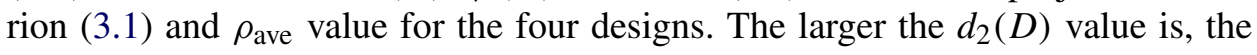
better a design is. For all other criteria, the smaller the better. As expected, each design is the best under the corresponding criterion. The uniform projection design $D_{4}$ performs well with respect to other criteria and is the best under the correlation criterion. Figure 1 displays bivariate projection plots of the four designs. The uniform projection design $D_{4}$ has the best projection properties among the four designs. Each grid has one point for $D_{4}$ whereas several grids have no points for the other three designs.

4. Projection properties. The following theorem shows that uniform projection designs have good space-filling properties not only in two dimensions, but also in all dimensions.

THEOREM 1. Let $D$ be a balanced $\left(n, s^{m}\right)$ design. For any $2 \leq k \leq m$,

$$
\frac{1}{\left(\begin{array}{c}
m \\
k
\end{array}\right)} \sum_{|u|=k} \phi\left(D_{u}\right)=\phi(D),
$$

where $D_{u}$ is the projected design onto $k$ factors indexed by $u$. 
TABLE 1

Four $25 \times 3$ LHDs

\begin{tabular}{|c|c|c|c|c|c|c|c|c|c|c|c|}
\hline \multicolumn{3}{|c|}{ Uniform $D_{1}$} & \multicolumn{3}{|c|}{$\operatorname{Maximin} D_{2}$} & \multicolumn{3}{|c|}{ MaxPro $D_{3}$} & \multicolumn{3}{|c|}{ UPD $D_{4}$} \\
\hline 18 & 16 & 14 & 0 & 2 & 20 & 0 & 6 & 12 & 2 & 3 & 2 \\
\hline 19 & 9 & 0 & 1 & 20 & 10 & 1 & 15 & 5 & 4 & 5 & 13 \\
\hline 11 & 1 & 2 & 2 & 7 & 12 & 2 & 18 & 21 & 0 & 11 & 9 \\
\hline 16 & 20 & 3 & 3 & 13 & 21 & 3 & 9 & 0 & 3 & 16 & 17 \\
\hline 20 & 22 & 12 & 4 & 9 & 3 & 4 & 12 & 17 & 1 & 22 & 22 \\
\hline 14 & 7 & 10 & 5 & 19 & 0 & 5 & 0 & 10 & 8 & 0 & 7 \\
\hline 4 & 17 & 1 & 6 & 23 & 19 & 6 & 21 & 3 & 6 & 8 & 19 \\
\hline 12 & 12 & 7 & 7 & 14 & 13 & 7 & 4 & 19 & 9 & 14 & 24 \\
\hline 10 & 15 & 24 & 8 & 0 & 7 & 8 & 23 & 13 & 5 & 18 & 4 \\
\hline 22 & 14 & 5 & 9 & 3 & 17 & 9 & 11 & 7 & 7 & 20 & 11 \\
\hline 2 & 21 & 22 & 10 & 21 & 8 & 10 & 14 & 24 & 12 & 2 & 21 \\
\hline 15 & 11 & 21 & 11 & 8 & 9 & 11 & 3 & 1 & 10 & 9 & 0 \\
\hline 1 & 5 & 4 & 12 & 6 & 1 & 12 & 8 & 15 & 14 & 12 & 14 \\
\hline 3 & 10 & 11 & 13 & 18 & 23 & 13 & 19 & 9 & 13 & 15 & 6 \\
\hline 23 & 3 & 8 & 14 & 15 & 2 & 14 & 1 & 22 & 11 & 24 & 15 \\
\hline 0 & 13 & 15 & 15 & 10 & 18 & 15 & 7 & 4 & 17 & 4 & 10 \\
\hline 8 & 23 & 6 & 16 & 24 & 16 & 16 & 16 & 18 & 15 & 7 & 5 \\
\hline 7 & 8 & 18 & 17 & 16 & 11 & 17 & 24 & 6 & 19 & 10 & 18 \\
\hline 9 & 4 & 13 & 18 & 1 & 15 & 18 & 13 & 11 & 16 & 19 & 20 \\
\hline 6 & 19 & 9 & 19 & 22 & 4 & 19 & 22 & 23 & 18 & 23 & 1 \\
\hline 24 & 18 & 19 & 20 & 4 & 6 & 20 & 2 & 14 & 21 & 1 & 16 \\
\hline 21 & 6 & 23 & 21 & 5 & 24 & 21 & 17 & 2 & 23 & 6 & 23 \\
\hline 13 & 24 & 17 & 22 & 12 & 5 & 22 & 10 & 20 & 22 & 13 & 3 \\
\hline 17 & 0 & 16 & 23 & 17 & 22 & 23 & 5 & 8 & 20 & 17 & 12 \\
\hline 5 & 2 & 20 & 24 & 11 & 14 & 24 & 20 & 16 & 24 & 21 & 8 \\
\hline
\end{tabular}

Theorem 1 shows that the average $\phi$ value of all $k$-factor projected designs is $\phi(D)$. Thus a uniform projection design tends to have small $\phi\left(D_{u}\right)$ values for all projections. Theorem 1 does not hold for the CD criterion defined in (2.3).

We compare projection properties of uniform projection designs with three other types of designs: uniform designs, maximin distance designs and maximum

TABLE 2

Comparison of the four LHDs in Table 1

\begin{tabular}{lccccc}
\hline Design & CD $\times \mathbf{~ 1 0 0 0}$ & $\boldsymbol{d}_{\mathbf{2}}(\boldsymbol{D})$ & $\boldsymbol{\psi}(\boldsymbol{D})$ & $\boldsymbol{\phi}(\boldsymbol{D}) \times \mathbf{1 0 0 0}$ & $\rho_{\text {ave }}(\boldsymbol{D})$ \\
\hline Uniform $D_{1}$ & 1.421 & 5.385 & 0.046 & 0.533 & 0.013 \\
Maximin $D_{2}$ & 2.091 & 8.246 & 0.062 & 0.751 & 0.037 \\
MaxPro $D_{3}$ & 1.840 & 6.403 & 0.043 & 0.666 & 0.079 \\
UPD $D_{4}$ & 1.534 & 6.164 & 0.047 & 0.528 & 0.008 \\
\hline
\end{tabular}


$D_{1}[1,2]$

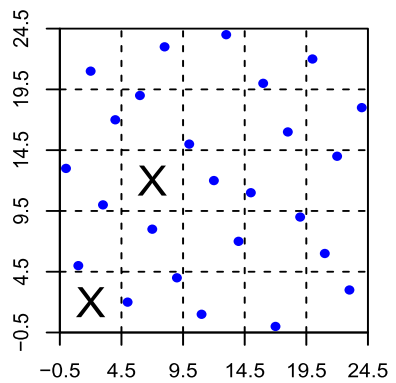

$D_{2}[1,2]$
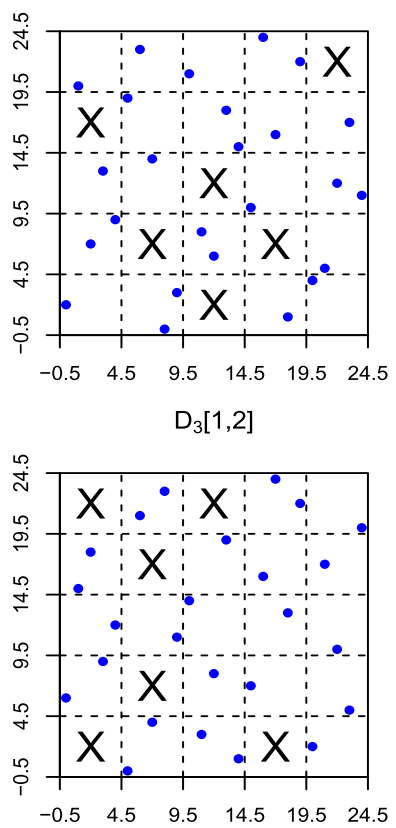

$\mathrm{D}_{4}[1,2]$

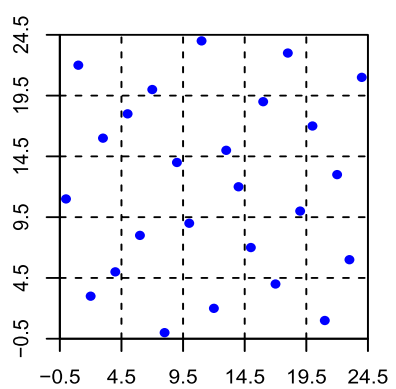

$D_{1}[1,3]$

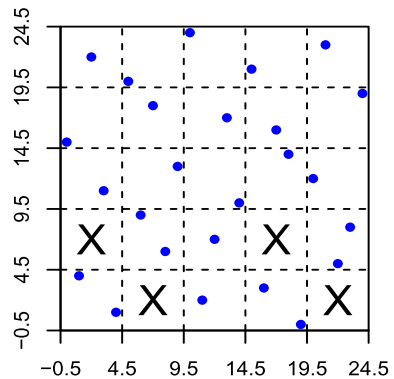

$\mathrm{D}_{2}[1,3]$

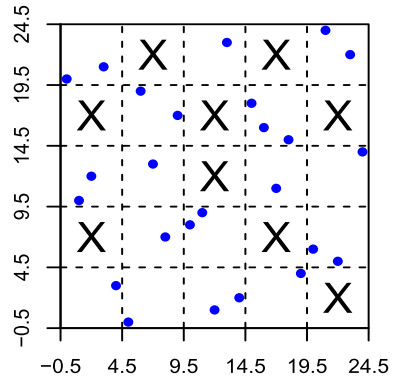

$D_{3}[1,3]$

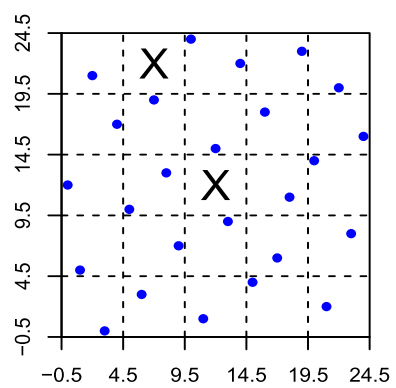

$D_{4}[1,3]$

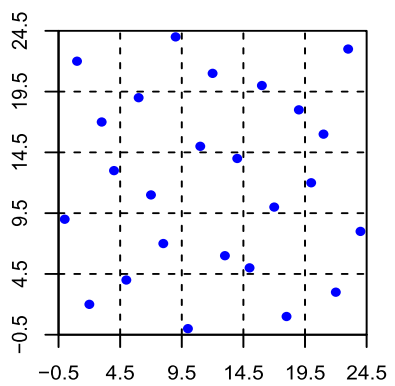

$D_{1}[2,3]$

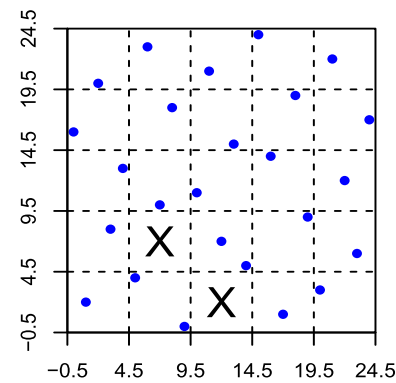

$D_{2}[2,3]$

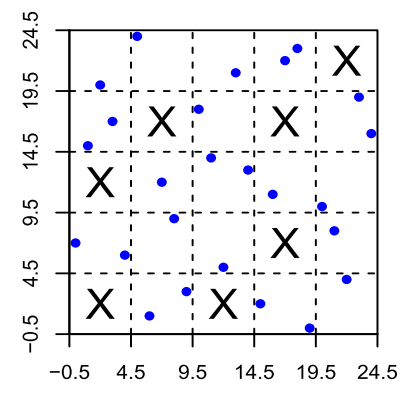

$D_{3}[2,3]$

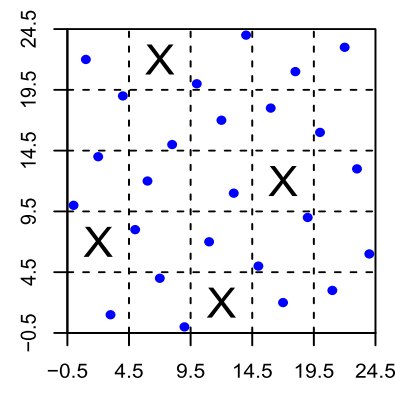

$\mathrm{D}_{4}[2,3]$

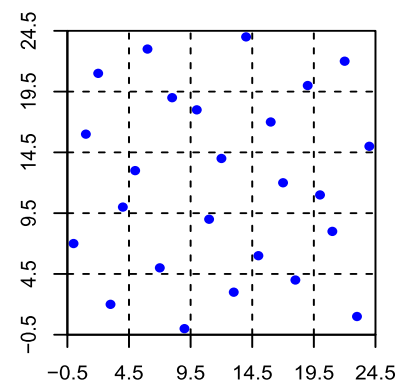

FIG. 1. Bivariate projections of four $25 \times 3$ LHDs in Table 1, where " $\mathrm{X}$ " means that there are no points in the grid and $D[i, j]$ stands for the projected design of $D$ onto the ith and $j$ th columns. 
(a)

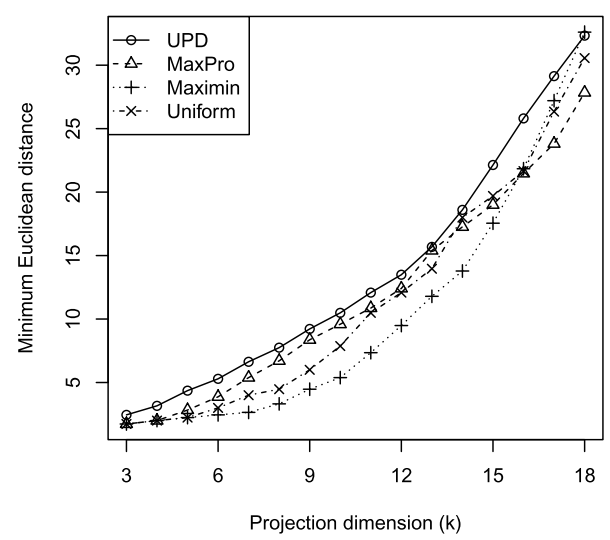

(c)

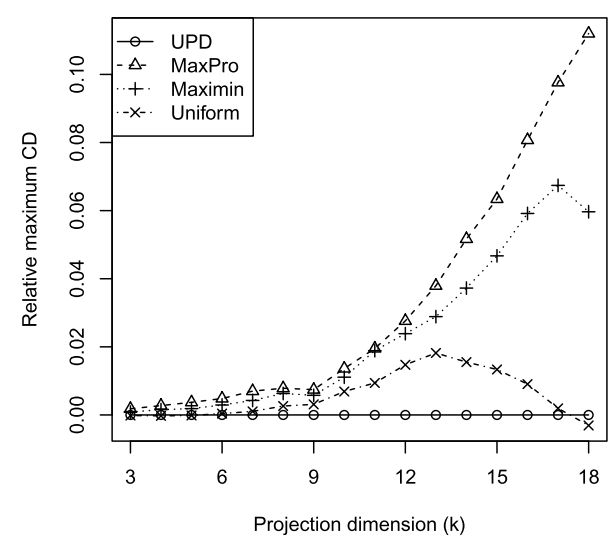

(b)

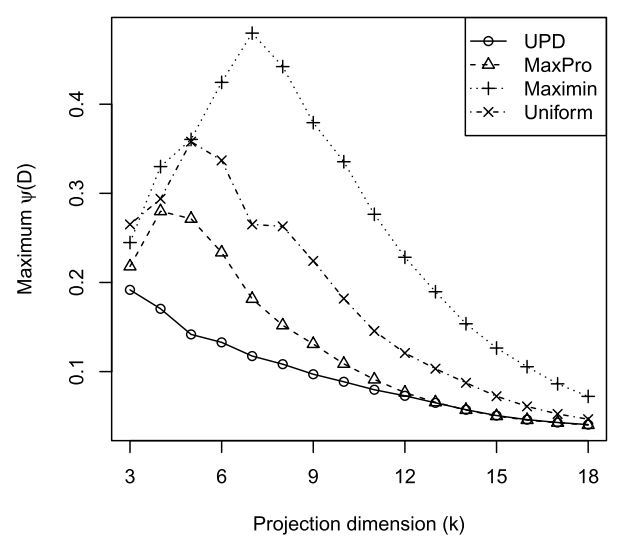

(d)

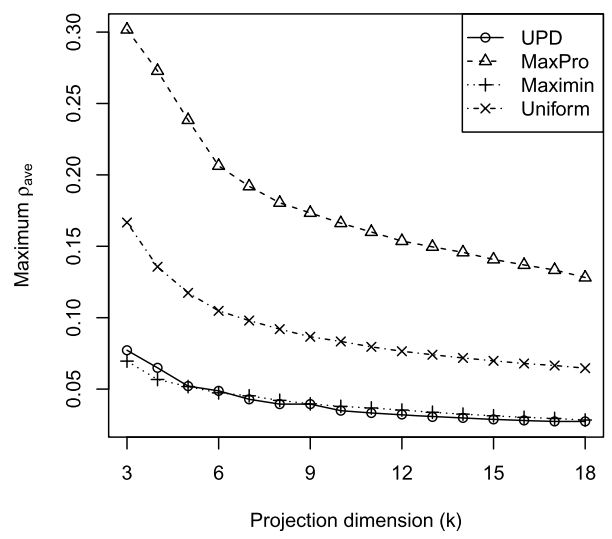

FIG. 2. Comparisons of projection designs under four criteria (a) minimum Euclidean distance (the larger the better), (b) maximum $\psi(D)$ (the smaller the better), (c) relative maximum $C D$ (the smaller the better) and (d) maximum $\rho_{\text {ave }}$ (the smaller the better).

projection designs. For illustration, we report the results for four $19 \times 18$ LHDs. The results of other sizes are similar. The uniform projection design is constructed in Example 3 in the next section. The $\phi$ values (multiplied by 1000) for the uniform projection design, uniform design, maximin design and maximum projection design are $1.478,1.482,1.543$ and 1.588 , respectively.

Figure 2 shows the comparisons of the projected designs onto $3 \leq k \leq 18$ dimensions. For each $k$, we evaluate all $\left(\begin{array}{c}m \\ k\end{array}\right)$ projected designs and determine the worst projection with respect to four criteria: (a) minimum Euclidean distance, (b) maximum $\psi(D)$ defined in $(2.2)$, (c) relative maximum centered $L_{2}$ discrepancy $(\mathrm{CD})$ and $(\mathrm{d})$ maximum correlation $\rho_{\text {ave }}$. The relative maximum CD value is computed as the difference of the $\mathrm{CD}$ values between the corresponding 
design and the uniform projection design. Figure 2 shows that our uniform projection design performs well for all dimensions under all criteria. Compared to the other three designs, the uniform projection design is more robust under various criteria.

5. Connection between the $\phi(D)$ criterion and distance criterion. Maximizing the minimum inter-site distance is a popular criterion for finding good space-filling designs. The $\mathrm{CD}(D)$ defined in (2.3) is a function of the pairwise Hamming distances between design points in $D$ for two-level designs [Fang and Mukerjee (2000)]; but this does not hold for general $s$-level designs [Tang, $\mathrm{Xu}$ and Lin (2012)]. For two-level designs, the Hamming distance is equivalent to the $L_{1}$ distance. It is a surprise that $\phi(D)$ defined in (3.1) is a function of the pairwise $L_{1}$-distances between design points in $D$ for general $s$-level designs.

THEOREM 2. For a balanced $\left(n, s^{m}\right)$ design $D=\left(x_{i k}\right)$,

$$
\phi(D)=\frac{g(D)}{4 m(m-1) n^{2} s^{2}}+C(m, s),
$$

where

$$
g(D)=\sum_{i=1}^{n} \sum_{j=1}^{n} d_{1}^{2}\left(x_{i}, x_{j}\right)-\frac{2}{n} \sum_{i=1}^{n}\left(\sum_{j=1}^{n} d_{1}\left(x_{i}, x_{j}\right)\right)^{2},
$$

$d_{1}\left(x_{i}, x_{j}\right)$ is defined in (2.1) with $p=1$, and

$$
C(m, s)=\frac{4(5 m-2) s^{4}+30(3 m-5) s^{2}+15 m+33}{720(m-1) s^{4}}+\frac{1+(-1)^{s}}{64 s^{4}} .
$$

The $\phi(D)$ defined in (3.1) measures the relationships of the columns whereas equation (5.2) measures the relationships of rows, so Theorem 2 establishes a link between the relationships of rows and columns of the design. In addition, Theorem 2 is important in the computational perspective as it provides a fast calculation of $\phi(D)$. The complexity for computing $\phi(D)$ based on (3.1) is $O\left(n^{2} m^{2}\right)$ whereas the complexity based on $(5.1)$ is $O\left(n^{2} m\right)$, the same complexity as for computing the maximin distance or the centered $L_{2}$-discrepancy without considering projections. Uniform projection designs can be constructed via search methods such as simulated annealing or threshold accepting algorithms in the same fashion as traditional maximin distance designs or uniform designs. We omit the details.

We now derive a lower bound of $\phi(D)$. By Theorem 2, $\phi(D)$ is a quadratic form of all pairwise $L_{1}$-distances between distinct rows. A major challenge here is that the quadratic form in (5.2) is not positive definite, and hence the traditional quadratic optimization technique cannot be applied directly. Intuitively, we should minimize $\sum_{i=1}^{n} \sum_{j=1}^{n} d_{1}^{2}\left(x_{i}, x_{j}\right)$ while maximizing $\sum_{i=1}^{n}\left(\sum_{j=1}^{n} d_{1}\left(x_{i}, x_{j}\right)\right)^{2}$, but these two objectives often contradict with each other. To derive a lower bound, we utilize the constraint that our designs are balanced; see the Appendix for details. 
THEOREM 3. For a balanced $\left(n, s^{m}\right)$ design $D, \phi(D) \geq L B$, where

$$
\begin{aligned}
L B= & \frac{5 m\left(4 s^{4}+2(13 n-17) s^{2}-n+5\right)-(n-1)\left(8 s^{4}+150 s^{2}-33\right)}{720(m-1)(n-1) s^{4}} \\
& +\frac{1+(-1)^{s}}{64 s^{4}} .
\end{aligned}
$$

The lower bound is achieved if and only if $D$ is an equidistant design under the $L_{1}$-distance.

Based on Theorem 3, we define the $\phi$-efficiency $\phi_{\text {eff }}(D)=L B / \phi(D)$ to measure the goodness of a design $D$. Wang, Xiao and Xu (2018) constructed a class of exact $L_{1}$-equidistant $n \times n$ LHDs whenever $2 n+1$ is an odd prime. By Theorem 3 , these are uniform projection designs with $\phi_{\mathrm{eff}}(D)=1$.

Now we give a construction method of uniform projection designs with $n$ runs and $n-1$ columns based on good lattice point designs for any odd prime $n$. Zhou and $\mathrm{Xu}$ (2015) studied space-filling properties of good lattice point designs and their linear permutations. Let $n$ be an odd prime and $D=\left(x_{i k}\right)$ be an $n \times(n-$ 1) good lattice point design, that is, $x_{i k}=(i \times k)(\bmod n)$. For an integer $b$, let $D_{b}=D+b=\left(x_{i k}+b\right)(\bmod n)$ be a linear permutation of $D$.

Wang, Xiao and $\mathrm{Xu}$ (2018) considered nonlinear permutations of good lattice point designs under the Williams transformation. The Williams transformation was first used by Williams (1949) to construct Latin square designs that are balanced for nearest neighbors. For any $x \in \mathcal{Z}_{n}=\{0,1, \ldots, n-1\}$, the Williams transformation is defined by

$$
W(x)= \begin{cases}2 x, & 0 \leq x<n / 2, \\ 2(n-x)-1, & n / 2 \leq x<n .\end{cases}
$$

The transformation defines a permutation of $\mathcal{Z}_{n}$. For example, when $n=7$, it permutes levels $(0,1,2,3,4,5,6)$ to $(0,2,4,6,5,3,1)$. Since $W(\cdot)$ is invertible, we use $W^{-1}(\cdot)$ to denote its inverse function. Let $E_{b}=W\left(D_{b}\right)$ be the Williams transformation of $D_{b}$, that is, $W\left(D_{b}\right)$ is an $n \times(n-1)$ matrix with the $(i, k)$ th entry being $W\left(\left(x_{i k}+b\right)(\bmod n)\right)$.

EXAMPLE 2. Let $n=19$. Table 3 shows the $\phi$ values and the corresponding $\phi$-efficiencies of $D_{b}$ and $E_{b}$ for $b=0,1, \ldots, 18$. From Table 3, several Williams transformed designs $E_{b}(b=2,7,11,12,16,17)$ have smaller $\phi$ values and are better than any linear permuted designs $D_{b}$.

The next theorem gives an analytical formula for computing $\phi\left(E_{b}\right)$ and the best choices of $b$ for minimizing $\phi\left(E_{b}\right)$ when $n$ is an odd prime. It also shows that the resulting designs are asymptotically optimal. 
TABLE 3

The $\phi$ values (multiplied by 1000) and $\phi$-efficiencies of $D_{b}$ and $E_{b}$ for $n=19$ and $b \in \mathcal{Z}_{n}$

\begin{tabular}{cccccccccc}
\hline $\boldsymbol{b}$ & $\boldsymbol{\phi}\left(\boldsymbol{D}_{\boldsymbol{b}}\right)$ & $\boldsymbol{\phi}_{\text {eff }}\left(\boldsymbol{D}_{\boldsymbol{b}}\right)$ & $\boldsymbol{\phi}\left(\boldsymbol{E}_{\boldsymbol{b}}\right)$ & $\boldsymbol{\phi}_{\text {eff }}\left(\boldsymbol{E}_{\boldsymbol{b}}\right)$ & $\boldsymbol{b}$ & $\boldsymbol{\phi}\left(\boldsymbol{D}_{\boldsymbol{b}}\right)$ & $\boldsymbol{\phi}_{\text {eff }}\left(\boldsymbol{D}_{\boldsymbol{b}}\right)$ & $\boldsymbol{\phi}\left(\boldsymbol{E}_{\boldsymbol{b}}\right)$ & $\boldsymbol{\phi}_{\text {eff }}\left(\boldsymbol{E}_{\boldsymbol{b}}\right)$ \\
\hline 0 & 2.107 & 0.696 & 2.641 & 0.555 & 10 & 1.662 & 0.882 & 1.989 & 0.738 \\
1 & 1.592 & 0.922 & 1.630 & 0.900 & 11 & 1.685 & 0.871 & 1.483 & 0.989 \\
2 & 1.703 & 0.861 & 1.478 & 0.992 & 12 & 1.757 & 0.835 & 1.555 & 0.943 \\
3 & 1.757 & 0.835 & 1.666 & 0.881 & 13 & 1.788 & 0.820 & 1.772 & 0.828 \\
4 & 1.773 & 0.828 & 1.847 & 0.794 & 14 & 1.773 & 0.828 & 1.873 & 0.783 \\
5 & 1.788 & 0.820 & 1.847 & 0.794 & 15 & 1.757 & 0.835 & 1.772 & 0.828 \\
6 & 1.757 & 0.835 & 1.666 & 0.881 & 16 & 1.703 & 0.861 & 1.555 & 0.943 \\
7 & 1.685 & 0.871 & 1.478 & 0.992 & 17 & 1.592 & 0.922 & 1.483 & 0.989 \\
8 & 1.662 & 0.882 & 1.630 & 0.900 & 18 & 2.107 & 0.696 & 1.989 & 0.738 \\
9 & 2.350 & 0.624 & 2.641 & 0.555 & & & & & \\
\hline
\end{tabular}

THEOREM 4. Let $n$ be an odd prime.

(i) For the $n \times(n-1) L H D E_{b}$, we have

$$
\phi\left(E_{b}\right)=L B+f^{2}(b) /\left[(n-2) n^{4}\right],
$$

where

$$
f(b)=(W(b)-(n-1) / 2)^{2}-\left(n^{2}-1\right) / 12
$$

and $L B=\left(12 n^{3}+154 n^{2}-12 n-29\right) /\left(720 n^{4}\right)$ is defined in Theorem 3 with $m=$ $n-1$ and $s=n$.

(ii) Let $c_{0}=\left\lfloor\sqrt{\left(n^{2}-1\right) / 12}\right\rfloor$ and $\lfloor x\rfloor$ be the integer part of $x$. Let

$$
c= \begin{cases}c_{0}, & c_{0} \geq \sqrt{\left(n^{2}-4\right) / 12}-1 / 2, \\ c_{0}+1, & c_{0}<\sqrt{\left(n^{2}-4\right) / 12}-1 / 2,\end{cases}
$$

and

$$
b^{*}=W^{-1}((n-1) / 2 \pm c) .
$$

Then $\phi\left(E_{b^{*}}\right)$ minimizes $\phi\left(E_{b}\right)$ among $b \in Z_{n}$.

(iii) The $\phi$-efficiency of $E_{b^{*}}$ is

$$
\phi_{\mathrm{eff}}\left(E_{b^{*}}\right)=\frac{L B}{\phi\left(E_{b^{*}}\right)}>\frac{n^{2}}{n^{2}+5} \rightarrow 1 \quad(n \rightarrow \infty) .
$$

EXAmple 3. Suppose $n=19$. Then $c_{0}=\left\lfloor\sqrt{\left(19^{2}-1\right) / 12}\right\rfloor=5$. We set $c=c_{0}=5$ based on Theorem 4(ii) because $c_{0} \geq \sqrt{\left(n^{2}-4\right) / 12}-1 / 2$. Then $b^{*}=W^{-1}(9 \pm 5)=2$ or 7 by (5.4). This agrees with Table 3. For these two designs, we have $\phi_{\text {eff }}\left(E_{b^{*}}\right)=0.992$. 
In general, for an odd prime $n \geq 23$, by (5.5), we have $\phi_{\text {eff }}\left(E_{b^{*}}\right)>n^{2} /\left(n^{2}+\right.$ 5) $>0.99$ when $b^{*}$ is chosen by (5.4). Wang, Xiao and $\mathrm{Xu}$ (2018) studied the performance of $E_{b}$ under the $L_{1}$-distance and correlation criteria. The optimal choices of $b$ are different under different criteria. Uniform projection designs $E_{b^{*}}$ always have large $L_{1}$-distance and small correlations.

6. Applications. We illustrate an application of uniform projection designs using a drug combination experiment on lung cancer conducted by Al-Shyoukh et al. (2011). The experiment used a 512-run and 8-level full factorial design to study three drugs for both normal cells and lung cancer cells. The response variable was the ATP level (standardized to 0-1 range) of the cell measured 72 hours after the drug treatments. One purpose of this experiment was to model the response surface and to systematically quantify the characterization of cellular responses.

Polynomial models, nonlinear Hill-based models and neural networks have been used to analyze drug combination experiments [Al-Shyoukh et al. (2011), Ning et al. (2014)]. Xiao, Wang and Xu (2017) recently proposed the use of Kriging models for drug combination experiments. Kriging models are widely used in computer experiments for optimization and sensitivity analysis. Different from deterministic simulations and computer experiments, combinatorial drug experiments have measurement errors. So Xiao, Wang and Xu (2017) considered a Kriging model with a noise term

$$
y(x)=\mu+Z(x)+\varepsilon,
$$

where $y(x)$ is the response at point $x, \mu$ is the intercept (or trend), $Z(x)$ is a Gaussian process with mean 0 , variance $\sigma^{2}$ and correlation function $R(\cdot)$ and $\varepsilon \sim$ $N\left(0, \tau^{2}\right)$ is independent of $Z(x)$. The correlation function is defined as

$$
R\left(x_{i}, x_{j}\right)=\operatorname{cor}\left(Z\left(x_{i}\right), Z\left(x_{j}\right)\right)=\prod_{k=1}^{m} K\left(\left|x_{i k}-x_{j k}\right| / \theta_{k}\right),
$$

where $m$ is the number of factors, $\theta_{k}$ are the range parameters and $K(\cdot)$ is the correlation kernel. Xiao, Wang and Xu (2017) recommended the use of Matérn kernel $K(h)=\left(1+\sqrt{5} h+5 h^{2} / 3\right) \exp (-\sqrt{5} h)$. Unknown parameters can be estimated by the maximum likelihood estimation method.

We consider four possible designs using the four $25 \times 3$ LHDs given in Table 1 . Since each factor has eight levels, we collapse the 25 levels into eight levels via a simple transformation, $x \rightarrow\lfloor 8 x / 25\rfloor$, and form the designs by selecting points from the original grids of 512 observations. Given an $n$-run design, we fit a model using $n$ observations and use the model to predict all 512 observations. Then we compute the mean square error (MSE) based on the 512 predicted and actual responses. Neural networks yield slightly different results each time so we report the average value from 100 trials.

Table 4 displays MSE (multiplied by 1000) for different models and designs for both normal and cancer cells. For comparison, we include the results from a 27 -run 
TABLE 4

MSE (multiplied by 1000) for different models and designs

(a) Normal cells

\begin{tabular}{|c|c|c|c|c|c|c|}
\hline & \multicolumn{4}{|c|}{ 25-Run designs } & \multicolumn{2}{|c|}{ Other designs } \\
\hline & Uniform & Maximin & MaxPro & UPD & $D_{27}$ & $D_{512}$ \\
\hline Kriging & 0.47 & 0.52 & 0.62 & 0.22 & 0.31 & 0.002 \\
\hline Neural network & 3.31 & 3.76 & 2.44 & 2.36 & 6.78 & 0.21 \\
\hline Polynomial & 2.20 & 1.18 & 3.22 & 0.74 & 1.12 & 0.48 \\
\hline Hill-based & 1.52 & 2.59 & 1.95 & 1.63 & 3.30 & 0.89 \\
\hline
\end{tabular}

(b) Cancer cells

\begin{tabular}{lcccccccc}
\hline & \multicolumn{4}{c}{ 25-Run designs } & & \multicolumn{2}{c}{ Other designs } \\
\cline { 2 - 3 } & Uniform & Maximin & MaxPro & UPD & & $\boldsymbol{D}_{\mathbf{2 7}}$ & $\boldsymbol{D}_{\mathbf{5 1 2}}$ \\
\hline Kriging & 1.78 & 0.63 & 1.87 & 0.21 & & 1.08 & 0.003 \\
Neural network & 3.60 & 4.16 & 3.34 & 3.31 & & 10.87 & 0.48 \\
Polynomial & 20.16 & 13.58 & 16.04 & & 4.42 & & 5.84 & 2.98 \\
Hill-based & 1.60 & 1.43 & 6.69 & & 2.23 & & 4.70 & 1.42 \\
\hline
\end{tabular}

3-level design $\left(D_{27}\right)$ from Xiao, Wang and $\mathrm{Xu}(2017)$ and the original 8-level full factorial design with 512 runs $\left(D_{512}\right)$.

Table 4 shows that Kriging models are always the best in prediction among the four types of models. Among the four 25-run designs, the uniform projection design (UPD) is the most robust for fitting various models, especially for cancer cells. The 25-run uniform projection design performs better than the 27-run 3-level design used by Xiao, Wang and Xu (2017) for all four types of models. Remarkably, the Kriging models using the 25-run uniform projection design have similar or smaller MSEs than neural network, polynomial and Hill-based models using all 512 runs. Uniform projection designs, having good projections in all dimensions, can provide accurate estimations and precise predictions with a small number of runs.

7. Concluding remarks. When only a subset of the input variables are active, uniformity of projected designs in low-dimensional spaces is important. We proposed a new design criterion which considers the projection uniformity under the centered $L_{2}$-discrepancy. We showed that uniform projection designs have good space-filling properties not only in two dimensions, but also in all dimensions. Uniform projection designs are robust and perform well under other design criteria. Besides, we linked the new criterion with pairwise $L_{1}$-distances between design points (Theorem 2 ) and showed that maximin $L_{1}$-equidistant designs are 
uniform projection designs (Theorem 3). We also constructed a class of asymptotically optimal uniform projection designs based on good lattice point designs and the Williams transformation. The theoretical results can be easily extended to other commonly used discrepancies such as wrap-around $L_{2}$-discrepancy. We further provided an application of uniform projection designs to a multidrug combination experiment. Uniform projection designs have good projection properties and are ideal for both computer and physical experiments. There are alternative approaches to constructing LHDs using orthogonal arrays or similar structures when lower dimensional properties are important; see Tang (1993), He and Tang (2013, 2014), He, Cheng and Tang (2018) and Xiao and Xu (2018) for details.

\section{APPENDIX: PROOFS}

Proof of TheOrem 1. From the definition of $\phi(D)$ in (3.1), for $2 \leq k \leq m$ and $|u|=k$, we have

$$
\phi\left(D_{u}\right)=\frac{1}{\left(\begin{array}{c}
k \\
2
\end{array}\right)} \sum_{|v|=2, v \subseteq u} \mathrm{CD}\left(D_{v}\right)
$$

Then

$$
\sum_{|u|=k} \phi\left(D_{u}\right)=\sum_{|u|=k} \frac{1}{\left(\begin{array}{c}
k \\
2
\end{array}\right)} \sum_{\substack{|v|=2 \\
v \subseteq u}} \operatorname{CD}\left(D_{v}\right)=\frac{1}{\left(\begin{array}{c}
k \\
2
\end{array}\right)} \sum_{\substack{|v|=2 \\
v \subseteq\{1, \ldots, m\}}}\left(\begin{array}{c}
m-2 \\
k-2
\end{array}\right) \operatorname{CD}\left(D_{v}\right) .
$$

Because $\left(\begin{array}{c}m \\ k\end{array}\right)\left(\begin{array}{l}k \\ 2\end{array}\right)=\left(\begin{array}{c}m-2 \\ k-2\end{array}\right)\left(\begin{array}{c}m \\ 2\end{array}\right)$, we have

$$
\frac{1}{\left(\begin{array}{c}
m \\
k
\end{array}\right)} \sum_{|u|=k} \phi\left(D_{u}\right)=\frac{1}{\left(\begin{array}{c}
m \\
2
\end{array}\right)} \sum_{\substack{|v|=2 \\
v \subseteq\{1, \ldots, m\}}} \mathrm{CD}\left(D_{v}\right)=\phi(D) .
$$

To prove Theorems 2 and 3, we need the following two lemmas.

LEMMA 1. For a balanced $\left(n, s^{m}\right)$ design $D=\left(x_{i k}\right)$ with levels from $\mathcal{Z}_{s}$, let $z_{i k}=\left(2 x_{i k}-s+1\right) /(2 s)$. For any $k=1, \ldots, m$,
(i) $\quad \sum_{i=1}^{n}\left|z_{i k}\right|= \begin{cases}n\left(s^{2}-1\right) /\left(4 s^{2}\right), & \text { s odd, } \\ n / 4, & \text { s even; }\end{cases}$
(ii) $\sum_{i=1}^{n}\left|z_{i k}\right|^{2}=n\left(s^{2}-1\right) /\left(12 s^{2}\right)$;
(iii) $\quad \sum_{i=1}^{n}\left|z_{i k}\right|^{3}= \begin{cases}n\left(s^{2}-1\right)^{2} /\left(32 s^{4}\right), & \text { s odd, } \\ n\left(s^{2}-2\right) /\left(32 s^{2}\right), & \text { s even; }\end{cases}$ 
(iv) $\sum_{i=1}^{n}\left|z_{i k}\right|^{4}=n\left(s^{2}-1\right)\left(3 s^{2}-7\right) /\left(240 s^{4}\right)$;

(v) $\sum_{i=1}^{n} \sum_{j=1}^{n}\left|z_{i k}-z_{j k}\right|^{p}=n^{2}\left(s^{2}-1\right) /\left(3 p s^{2}\right), \quad p=1,2$;

(vi) $\sum_{i=1}^{n} \sum_{j=1}^{n}\left|z_{j k}\right|\left|z_{i k}-z_{j k}\right|= \begin{cases}3 n^{2}\left(s^{2}-1\right)^{2} /\left(32 s^{4}\right), & s \text { odd, } \\ n^{2}\left(3 s^{2}-4\right) /\left(32 s^{2}\right), & \text { s even. }\end{cases}$

Lemma 1 can be proved via some tedious calculations so we omit the details.

LEMMA 2. Let $D=\left(x_{i k}\right)_{n \times m}$ be a balanced $\left(n, s^{m}\right)$ design with levels from $\mathcal{Z}_{s}$. For any $i=1, \ldots, n$,

$$
\sum_{j=1}^{n} d_{1}\left(x_{i}, x_{j}\right)=\frac{n m\left(s^{2}-1\right)}{4 s}+\frac{n}{s} d_{2}^{2}\left(x_{i}, s_{0}\right),
$$

where $x_{i}$ is the ith row of $D, d_{1}\left(x_{i}, x_{j}\right)$ is defined in (2.1) with $p=1, s_{0}=(s-$ 1) $/ 2$, and $d_{2}\left(x_{i}, s_{0}\right)=\left(\sum_{k=1}^{m}\left(x_{i k}-s_{0}\right)^{2}\right)^{1 / 2}$.

PROOF. Because each column of $D$ contains each of the $s$ levels exactly $n / s$ times, we have

$$
\begin{aligned}
\sum_{j=1}^{n} d_{1}\left(x_{i}, x_{j}\right) & =\sum_{j=1}^{n} \sum_{k=1}^{m}\left|x_{j k}-x_{i k}\right|=\sum_{k=1}^{m} \sum_{j=1}^{n}\left|x_{j k}-x_{i k}\right| \\
& =\sum_{k=1}^{m} \frac{n}{s}\left[\left(1+\cdots+\left|(s-1)-x_{i k}\right|\right)+\left(0+1+\cdots+\left|x_{i k}\right|\right)\right] \\
& =\sum_{k=1}^{m} \frac{n}{2 s}\left[\left(s-1-x_{i k}\right)^{2}+\left(s-1-x_{i k}\right)+\left(x_{i k}\right)^{2}+x_{i k}\right] \\
& =\frac{n m\left(s^{2}-1\right)}{4 s}+\frac{n}{s} \sum_{k=1}^{m}\left(x_{i k}-(s-1) / 2\right)^{2} .
\end{aligned}
$$

This completes the proof.

Proof of TheOREM 2. For any balanced $\left(n, s^{m}\right)$ design $D=\left(x_{i k}\right)$, let $Z=\left(z_{i k}\right)$ where $z_{i k}=\left(2 x_{i k}-s+1\right) /(2 s)$. Let $f_{k}^{i}=1+\frac{1}{2}\left|z_{i k}\right|-\frac{1}{2}\left|z_{i k}\right|^{2}$ and $d_{p}\left(z_{i}, 0\right)=\left(\sum_{k=1}^{m}\left|z_{i k}\right|^{p}\right)^{1 / p}$ for $p=1$ and 2. By Lemma $1, \sum_{i=1}^{n}\left(f_{k}^{i}\right)^{2}$ is a constant independent of $k$. Then

$$
G_{1}=\sum_{i=1}^{n} \sum_{1 \leq k_{1}<k_{2} \leq m} f_{k_{1}}^{i} f_{k_{2}}^{i}
$$




$$
\begin{aligned}
& =\sum_{i=1}^{n} \frac{1}{2}\left\{\left(f_{1}^{i}+\cdots+f_{m}^{i}\right)^{2}-\left(\left(f_{1}^{i}\right)^{2}+\cdots+\left(f_{m}^{i}\right)^{2}\right)\right\} \\
& =\frac{1}{2} \sum_{i=1}^{n}\left(m+\frac{1}{2} d_{1}\left(z_{i}, 0\right)-\frac{1}{2} d_{2}^{2}\left(z_{i}, 0\right)\right)^{2}-\frac{m}{2} \sum_{i=1}^{n}\left(f_{1}^{i}\right)^{2} \\
& =\frac{1}{8} \sum_{i=1}^{n}\left(d_{1}\left(z_{i}, 0\right)-d_{2}^{2}\left(z_{i}, 0\right)\right)^{2}+C_{1}(n, m, s),
\end{aligned}
$$

where

$$
C_{1}(n, m, s)=\frac{n m^{2}}{2}+\frac{m}{2} \sum_{i=1}^{n}\left(d_{1}\left(z_{i}, 0\right)-d_{2}^{2}\left(z_{i}, 0\right)\right)-\frac{m}{2} \sum_{i=1}^{n}\left(f_{1}^{i}\right)^{2}
$$

is a constant by Lemma 1 .

Let $g_{k}^{i j}=1+\frac{1}{2}\left|z_{i k}\right|+\frac{1}{2}\left|z_{j k}\right|-\frac{1}{2}\left|z_{i k}-z_{j k}\right|$. By Lemma $1, \sum_{i=1}^{n} \sum_{j=1}^{n}\left(g_{k}^{i j}\right)^{2}$ is a constant independent of $k$. Then

$$
\begin{aligned}
G_{2} & =\sum_{i=1}^{n} \sum_{j=1}^{n} \sum_{1 \leq k_{1}<k_{2} \leq m} g_{k_{1}}^{i j} g_{k_{2}}^{i j} \\
& =\frac{1}{2} \sum_{i=1}^{n} \sum_{j=1}^{n}\left\{\left(g_{1}^{i j}+\cdots+g_{m}^{i j}\right)^{2}-\left(\left(g_{1}^{i j}\right)^{2}+\cdots+\left(g_{m}^{i j}\right)^{2}\right)\right\} \\
& =\frac{1}{2} \sum_{i=1}^{n} \sum_{j=1}^{n}\left(m+\frac{1}{2} d_{1}\left(z_{i}, 0\right)+\frac{1}{2} d_{1}\left(z_{j}, 0\right)-\frac{1}{2} d_{1}\left(z_{i}, z_{j}\right)\right)^{2}-\frac{m}{2} \sum_{i=1}^{n} \sum_{j=1}^{n}\left(g_{1}^{i j}\right)^{2} \\
& =\frac{1}{8} \sum_{i=1}^{n} \sum_{j=1}^{n}\left(d_{1}\left(z_{i}, 0\right)+d_{1}\left(z_{j}, 0\right)-d_{1}\left(z_{i}, z_{j}\right)\right)^{2}+C_{2}(n, m, s),
\end{aligned}
$$

where

$$
\begin{aligned}
C_{2}(n, m, s)= & \frac{n^{2} m^{2}}{2}+m \sum_{i=1}^{n} \sum_{j=1}^{n} d_{1}\left(z_{i}, 0\right)-\frac{m}{2} \sum_{i=1}^{n} \sum_{j=1}^{n} d_{1}\left(z_{i}, z_{j}\right) \\
& -\frac{m}{2} \sum_{i=1}^{n} \sum_{j=1}^{n}\left(g_{1}^{i j}\right)^{2}
\end{aligned}
$$

is a constant by Lemma 1 .

Let $s_{0}=(s-1) / 2$. Since $d_{1}\left(z_{i}, 0\right)=d_{1}\left(x_{i}, s_{0}\right) / s, d_{2}\left(z_{i}, 0\right)=d_{2}\left(x_{i}, s_{0}\right) / s$, and $d_{1}\left(z_{i}, z_{j}\right)=d_{1}\left(x_{i}, x_{j}\right) / s$, we have

$$
\phi(D)=\frac{2}{m(m-1) n^{2}} G_{2}-\frac{4}{m(m-1) n} G_{1}+\left(\frac{13}{12}\right)^{2}
$$




$$
\begin{aligned}
= & \frac{1}{4 n^{2} m(m-1) s^{2}} \sum_{i=1}^{n} \sum_{j=1}^{n}\left(d_{1}\left(x_{i}, s_{0}\right)+d_{1}\left(x_{j}, s_{0}\right)-d_{1}\left(x_{i}, x_{j}\right)\right)^{2} \\
& -\frac{1}{2 m(m-1) n s^{2}} \sum_{i=1}^{n}\left(d_{1}\left(x_{i}, s_{0}\right)-d_{2}^{2}\left(x_{i}, s_{0}\right) / s\right)^{2}+C_{3}(n, m, s),
\end{aligned}
$$

where

$$
C_{3}(n, m, s)=\frac{2}{m(m-1) n^{2}} C_{2}(n, m, s)-\frac{4}{m(m-1) n} C_{1}(n, m, s)+\left(\frac{13}{12}\right)^{2} .
$$

Then the result follows from Lemmas 1 and 2 and some tedious algebra.

To prove Theorem 3, we introduce some notation. Let $I_{n}$ be the identity matrix of order $n, 0_{n}$ a column vector of $n$ zeros, $1_{n}$ a column vector of $n$ ones and $J_{n}=1_{n} 1_{n}^{\mathrm{T}}$, where $1_{n}^{\mathrm{T}}$ is the transpose of $1_{n}$.

Let $f_{n}=n(n-1) / 2$ and $B_{2}=(1,1)^{\mathrm{T}}$. For $n>2$, define $n \times f_{n}$ matrix $B_{n}$ recursively as follows:

$$
B_{n}=\left(\begin{array}{ll}
1_{n-1}^{\mathrm{T}} & 0_{f_{n-1}}^{\mathrm{T}} \\
I_{n-1} & B_{n-1}
\end{array}\right)
$$

and $A_{n}=I_{f_{n}}-\frac{1}{n} B_{n}^{\mathrm{T}} B_{n}$.

\section{LEMMA 3.}

(i) $B_{n} 1_{f_{n}}=(n-1) 1_{n}$ and $B_{n}^{\mathrm{T}} 1_{n}=2 \times 1_{f_{n}}$;

(ii) $B_{n} B_{n}^{\mathrm{T}}=(n-2) I_{n}+J_{n}$;

(iii) The eigenvalues of $A_{n}$ are $1,2 / n$ and $(2-n) / n$ with multiplicities of $f_{n}-$ $n, n-1$ and 1 , respectively.

(iv) For $n>2, A_{n}$ is invertible and $A_{n}^{-1} 1_{f_{n}}=\frac{n}{2-n} 1_{f_{n}}$.

PROOF. Parts (i) and (ii) can be obtained by the definition of $B_{n}$ directly.

(iii) There exists a one-to-one correspondence between the eigenvalues of $A_{n}$ and the eigenvalues of $B_{n}^{\mathrm{T}} B_{n}$, that is, if $\mu$ is an eigenvalue of $B_{n}^{\mathrm{T}} B_{n}$, then $1-\mu / n$ must be an eigenvalue of $A_{n}$. Hence we only need to derive the eigenvalues of $B_{n}^{\mathrm{T}} B_{n}$. From (ii), we know that $B_{n} B_{n}^{\mathrm{T}}=(n-2) I_{n}+J_{n}$. It is straightforward to show that $B_{n} B_{n}^{\mathrm{T}}$ has eigenvalues $n-2$ and $2 n-2$ with multiplicities of $n-1$ and 1 , respectively. Therefore, $B_{n}^{\mathrm{T}} B_{n}$ has eigenvalues $0, n-2$, and $2 n-2$ with multiplicities of $f_{n}-n, n-1$ and 1 , respectively.

(iv) For $n>2, A_{n}$ is invertible following part (iii). In addition, $A_{n} 1_{f_{n}}=\frac{2-n}{n} 1_{f_{n}}$, and hence $A_{n}^{-1} 1_{f_{n}}=\frac{n}{2-n} 1_{f_{n}}$. This completes the proof.

Proof of TheOREM 3. Let $y$ be the $f_{n}$-dimensional column vector defined by

$$
y=\left(d_{1}\left(x_{1}, x_{2}\right), \ldots, d_{1}\left(x_{1}, x_{n}\right), d_{1}\left(x_{2}, x_{3}\right), \ldots, d_{1}\left(x_{n-1}, x_{n}\right)\right)^{\mathrm{T}},
$$


that is, $y$ collects all $d_{1}\left(x_{i}, x_{j}\right)$ 's for $i<j$ according to the lexicographic order, where $f_{n}=n(n-1) / 2$. Due to the symmetry $d_{1}\left(x_{i}, x_{j}\right)=d_{1}\left(x_{j}, x_{i}\right)$, it is easy to verify that the $i$ th element of $B_{n} y$ is $\sum_{j=1}^{n} d_{1}\left(x_{i}, x_{j}\right)$, where $B_{n}$ is defined in (A.1). Then we have

$$
g(D)=2 y^{\mathrm{T}} y-\frac{2}{n} y^{\mathrm{T}} B_{n}^{\mathrm{T}} B_{n} y=2 y^{\mathrm{T}} A_{n} y .
$$

To simplify the problem, we enlarge the domain of $y$ to be $\mathbb{R}_{+}^{f_{n}}=\left\{y_{i} \geq 0, i=\right.$ $\left.1, \ldots, f_{n}\right\}$ instead of a discrete space. Because $A_{n}$ is not positive definite, there is no global minimum. For balanced designs, by Lemma 1(v), we have the constraint $\sum_{i=1}^{n} \sum_{j=1}^{n} d_{1}\left(x_{i}, x_{j}\right)=m n^{2}\left(s^{2}-1\right) /(3 s)$. Then our problem is to minimize

$$
y^{\mathrm{T}} A_{n} y \quad \text { subject to } \quad y^{\mathrm{T}} 1_{f_{n}}=\frac{m n^{2}\left(s^{2}-1\right)}{6 s} \quad \text { for } y \in \mathbb{R}_{+}^{f_{n}} .
$$

Let $L=y^{\mathrm{T}} A_{n} y+\lambda\left(y^{\mathrm{T}} 1_{f_{n}}-\frac{m n^{2}\left(s^{2}-1\right)}{6 s}\right)$. Taking derivatives with respect to $y$ and $\lambda$, respectively, and setting them to 0 , we have

$$
\begin{aligned}
& \frac{\partial L}{\partial y}=2 A_{n} y+\lambda 1_{f_{n}}=0, \\
& \frac{\partial L}{\partial \lambda}=y^{\mathrm{T}} 1_{f_{n}}-\frac{m n^{2}\left(s^{2}-1\right)}{6 s}=0 .
\end{aligned}
$$

The equations can be solved based on Lemma 3, that is,

$$
y^{*}=\frac{m n\left(s^{2}-1\right)}{3 s(n-1)} 1_{f_{n}} \quad \text { and } \quad \lambda^{*}=\frac{2(n-2) m\left(s^{2}-1\right)}{3 s(n-1)} .
$$

To prove this solution is a strict minimum of the Lagrange multiplier problem, we perform the second derivative test. Let

$$
H=\left.\left(\frac{\partial^{2} L}{\partial(\lambda, y) \partial(\lambda, y)^{\mathrm{T}}}\right)\right|_{\left(\lambda^{*}, y^{*}\right)}=\left(\begin{array}{cc}
0 & -1_{f_{n}}^{\mathrm{T}} \\
-1_{f_{n}} & A_{n}
\end{array}\right)
$$

be the bordered Hessian matrix of $L$, where all of the partial derivatives are evaluated at $\lambda=\lambda^{*}$ and $y=y^{*}$. By Theorem 5.4 of Sundaram (1996), we only need to show that

$$
y^{\mathrm{T}} A_{n} y>0 \quad \text { for any } y \in \mathcal{N}=\left\{z \in \mathbb{R}^{f_{n}}: z^{\mathrm{T}} 1_{f_{n}}=0 \text { and } z \neq 0_{f_{n}}\right\} .
$$

Then the solution is a strict minimum.

Let $\lambda_{1}, \ldots, \lambda_{f_{n}}$ be the eigenvalues of $A_{n}$ and unit vectors $v_{1}, \ldots, v_{f_{n}}$ be the mutually orthogonal eigenvectors of $A_{n}$. Then any $y \in \mathcal{N}$ is a linear combination 
of vectors $v_{1}, \ldots, v_{f_{n}}$, that is,

$$
y=b_{1} v_{1}+\cdots+b_{f_{n}} v_{f_{n}} .
$$

By Lemma 3(iii), $A_{n}$ has one negative eigenvalue. Without loss of generality, let $\lambda_{1}=(2-n) / n$ be the unique negative eigenvalue. By Lemma 3(iv), $v_{1}=1_{f_{n}}$, so $b_{1}=y^{\mathrm{T}} v_{1}=y^{\mathrm{T}} 1_{f_{n}}=0$. Then

$$
y^{\mathrm{T}} A_{n} y=\lambda_{2} b_{2}^{2}+\cdots+\lambda_{f_{n}} b_{f_{n}}^{2}>0
$$

owing to $\lambda_{i}>0$ for $2 \leq i \leq f_{n}$.

In summary, $L$ achieves the minimum if and only if all the entries of $y$ are equal, which is equivalent to that $D$ is an equidistant design under the $L_{1}$-distance. It is easy to verify that the minimum is $L B$ as stated in the theorem.

We need the following lemma from Wang, Xiao and Xu (2018) to prove Theorem 4.

LEMMA 4. Let $n$ be an odd prime and $b \in \mathcal{Z}_{n}$. The $L_{1}$-distances of distinct rows of $E_{b}$ take on only three values,

$$
d_{i j}\left(E_{b}\right)= \begin{cases}\left(n^{2}-1\right) / 3+f(b), & i=n \text { or } j=n \\ \left(n^{2}-1\right) / 3-2 f(b), & i=n-j, \\ \left(n^{2}-1\right) / 3, & \text { otherwise }\end{cases}
$$

where $f(b)=(W(b)-(n-1) / 2)^{2}-\left(n^{2}-1\right) / 12$ and $d_{i j}\left(E_{b}\right)$ denotes the $L_{1}$ distance between the $i$ th and $j$ th rows of $E_{b}, i, j=1, \ldots, n$ and $i \neq j$.

Proof of TheOrem 4. (i) Using Lemma 4 and after some tedious algebra, we have

$$
g\left(E_{b}\right)=(n-1)\left\{36 f(b)^{2}-(n-2)\left(n^{2}-1\right)^{2}\right\} /\left(9 n^{2}\right) .
$$

Then the result follows from (5.1) and some additional algebra.

(ii) By (5.3), minimizing $\phi\left(E_{b}\right)$ is equivalent to minimizing $|f(b)|=\mid(W(b)-$ $(n-1) / 2)^{2}-\left(n^{2}-1\right) / 12 \mid$. Since $W(b)$ is an integer and $n$ is odd, the minimum value of $f(b)$ must be one of the two values, $c_{0}^{2}-\left(n^{2}-1\right) / 12$ or $\left(c_{0}+1\right)^{2}-\left(n^{2}-\right.$ 1)/12. Comparing these two values, we further have

$$
f\left(b^{*}\right)= \begin{cases}c_{0}^{2}-\left(n^{2}-1\right) / 12, & c_{0}^{2}+\left(c_{0}+1\right)^{2} \geq\left(n^{2}-1\right) / 6 \\ \left(c_{0}+1\right)^{2}-\left(n^{2}-1\right) / 12, & c_{0}^{2}+\left(c_{0}+1\right)^{2}<\left(n^{2}-1\right) / 6\end{cases}
$$

Then equation (5.4) follows after some algebra.

(iii) By part (i), we have

$$
\phi_{\mathrm{eff}}\left(E_{b^{*}}\right)=\left(1+\frac{720 f^{2}\left(b^{*}\right)}{(n-2)\left(12 n^{3}+154 n^{2}-12 n-29\right)}\right)^{-1} .
$$


By (5.4) and noting that $c_{0} \leq \sqrt{\left(n^{2}-1\right) / 12}<c_{0}+1$, we have that if $c_{0} \geq$ $\sqrt{\left(n^{2}-4\right) / 12}-1 / 2$, then

$$
0 \leq f\left(b^{*}\right)^{2}=\left[c_{0}^{2}-\left(n^{2}-1\right) / 12\right]^{2} \leq\left(n^{2}-4\right) / 12 .
$$

Similarly, if $c_{0}<\sqrt{\left(n^{2}-4\right) / 12}-1 / 2$, we also have

$$
0 \leq f\left(b^{*}\right)^{2}=\left[\left(c_{0}+1\right)^{2}-\left(n^{2}-1\right) / 12\right]^{2} \leq\left(n^{2}-4\right) / 12 .
$$

Substituting the inequality $f\left(b^{*}\right)^{2} \leq\left(n^{2}-4\right) / 12$ into (A.2) and using the fact that $n \geq 3$, we get

$$
\begin{aligned}
\phi_{\mathrm{eff}}\left(E_{b^{*}}\right) & \geq\left(1+\frac{60(n+2)}{12 n^{3}+154 n^{2}-12 n-29}\right)^{-1} \\
& >\left(1+\frac{60(n+2)}{12 n^{3}+24 n^{2}}\right)^{-1}=\frac{n^{2}}{n^{2}+5},
\end{aligned}
$$

which goes to one as $n \rightarrow \infty$. This completes the proof.

Acknowledgments. The research was done when the first two authors were visiting the Department of Statistics at University of California, Los Angeles. The authorship is in alphabetical order. The authors thank an Editor, an Associate Editor and two reviewers for their helpful comments.

\section{REFERENCES}

Al-Shyoukh, I., Yu, F., Feng, J., Yan, K., Dubinett, S., Ho, C. M., Shamma, J. S. and SUN, R. (2011). Systematic quantitative characterization of cellular responses induced by multiple signals. BMC Syst. Biol. 588.

BA, S., Myers, W. R. and Brenneman, W. A. (2015). Optimal sliced Latin hypercube designs. Technometrics 57 479-487. MR3425485

FAng, K.-T., LI, R. and SudJiAnto, A. (2006). Design and Modeling for Computer Experiments. Chapman \& Hall/CRC, Boca Raton, FL. MR2223960

FANG, K.-T. and MUKERJEE, R. (2000). A connection between uniformity and aberration in regular fractions of two-level factorials. Biometrika 87 193-198. MR1766839

FAnG, K.-T., Lin, D. K. J., Winker, P. and Zhang, Y. (2000). Uniform design: Theory and application. Technometrics 42 237-248. MR1801031

He, Y., Cheng, C.-S. and TAng, B. (2018). Strong orthogonal arrays of strength two plus. Ann. Statist. 46 457-468. MR3782373

HE, Y. and TANG, B. (2013). Strong orthogonal arrays and associated Latin hypercubes for computer experiments. Biometrika 100 254-260. MR3034340

HE, Y. and TANG, B. (2014). A characterization of strong orthogonal arrays of strength three. Ann. Statist. 42 1347-1360. MR3226159

HiCKERNELL, F. J. and LiU, M.-Q. (2002). Uniform designs limit aliasing. Biometrika 89 893-904. MR1946518

Johnson, M. E., Moore, L. M. and Ylvis aker, D. (1990). Minimax and maximin distance designs. J. Statist. Plann. Inference 26 131-148. MR1079258 
Joseph, V. R., GuL, E. and BA, S. (2015). Maximum projection designs for computer experiments. Biometrika 102 371-380. MR3371010

JosePh, V. R. and Hung, Y. (2008). Orthogonal-maximin Latin hypercube designs. Statist. Sinica 18 171-186. MR2416907

KleiJnen, J. P. (2017). Design and analysis of simulation experiments: Tutorial. In Advances in Modeling and Simulation (A. Tolk, J. Fowler, G. Shao and E. Yucesan, eds.) 135-158. Springer, New York.

LiANG, Y. Z., FANG, K. T. and XU, Q. S. (2001). Uniform design and its applications in chemistry and chemical engineering. Chemom. Intell. Lab. Syst. 58 43-57.

LIN, C. D. and TANG, B. (2015). Latin hypercubes and space-filling designs. In Handbook of Design and Analysis of Experiments (A. Dean, M. Morris, J. Stufken and D. Bingham, eds.) 593-625. CRC Press, Boca Raton, FL. MR3699363

MA, C.-X., FANG, K.-T. and LIN, D. K. J. (2003). A note on uniformity and orthogonality. J. Statist. Plann. Inference 113 323-334. MR1963050

Moon, H., DeAn, A. and SANTNER, T. (2011). Algorithms for generating maximin Latin hypercube and orthogonal designs. J. Stat. Theory Pract. 5 81-98. MR2829824

Moon, H., DeAn, A. M. and SANTNER, T. J. (2012). Two-stage sensitivity-based group screening in computer experiments. Technometrics 54 376-387. MR3006387

Ning, S., XU, H., Al-Shyoukh, I., Feng, J. and Sun, R. (2014). An application of a Hill-based response surface model for a drug combination experiment on lung cancer. Stat. Med. 33 42274236. MR3267406

Owen, A. B. (1994). Controlling correlations in Latin hypercube samples. J. Amer. Statist. Assoc. 89 1517-1522.

Santner, T. J., Williams, B. J. and NotZ, W. I. (2003). The Design and Analysis of Computer Experiments. Springer, New York. MR2160708

SUN, F. and TANG, B. (2017). A general rotation method for orthogonal Latin hypercubes. Biometrika 104 465-472. MR3698266

Sundaram, R. K. (1996). A First Course in Optimization Theory. Cambridge Univ. Press, Cambridge. MR1402910

TANG, B. (1993). Orthogonal array-based Latin hypercubes. J. Amer. Statist. Assoc. 88 1392-1397. MR1245375

TANG, B. (1998). Selecting Latin hypercubes using correlation criteria. Statist. Sinica 8 965-977. MR1651518

TANG, Y., XU, H. and Lin, D. K. J. (2012). Uniform fractional factorial designs. Ann. Statist. 40 891-907. MR2985937

UNIFORM DESIGN WEBSITE. http://www.math.hkbu.edu.hk/UniformDesign/.

WANG, L., XIAO, Q. and XU, H. (2018). Optimal maximin $L_{1}$-distance Latin hypercube designs based on good lattice point designs. Ann. Statist. 46 3741-3766. MR3852667

WANG, Y., YANG, J. and XU, H. (2018). On the connection between maximin distance designs and orthogonal designs. Biometrika 105 471-477. DOI:10.1093/biomet/asy005.

WiLliams, E. J. (1949). Experimental designs balanced for the estimation of residual effects of treatments. Aust. J. Sci. Res., Ser. A 2 149-168. MR0033508

Woods, D. C. and LEwIS, S. M. (2016). Design of experiments for screening. In Handbook of Uncertainty Quantification (R. Ghanem, D. Higdon and H. Owhadi, eds.) 1143-1185. Springer, New York.

XIAO, Q., WANG, L. and XU, H. (2017). Application of Kriging models for a drug combination experiment on lung cancer. Manuscript.

XIAO, Q. and XU, H. (2017). Construction of maximin distance Latin squares and related Latin hypercube designs. Biometrika 104 455-464. MR3698265

XIAO, Q. and XU, H. (2018). Construction of maximin distance designs via level permutation and expansion. Statist. Sinica. 28 1395-1414. MR3821010 
YE, K. Q. (1998). Orthogonal column Latin hypercubes and their application in computer experiments. J. Amer. Statist. Assoc. 93 1430-1439. MR1666638

ZHOU, Y.-D. and XU, H. (2014). Space-filling fractional factorial designs. J. Amer. Statist. Assoc. 109 1134-1144. MR3265686

ZHOU, Y. and XU, H. (2015). Space-filling properties of good lattice point sets. Biometrika 102 959-966. MR3431565

\section{F. SUN}

DEPARTMENT OF STATISTICS

KLAS AND SCHOOL OF MATHEMATICS AND STATISTICS

NORTHEAST NORMAL UNIVERSITY

ChangChun, Jilin 130024

CHINA

E-MAIL: sunfs359@nenu.edu.cn
Y. WANG

SCHOOL OF STATISTICS

EAST CHINA NORMAL UNIVERSITY

SHANGHAI 200241

CHINA

E-MAIL: wang41@163.com

\section{H. XU}

DEPARTMENT OF STATISTICS

UNIVERSITY OF CALIFORNIA, LOS ANGELES

8125 Math SCIENCES BLDG.

Los ANGeles, CALIFornia 90095-1554

USA

E-MAIL: hqxu@stat.ucla.edu 